\title{
TP53 co-mutations as an independent prognostic factor in 2nd and further line therapy-EGFR mutated non-small cell lung cancer IV patients treated with osimertinib
}

\author{
Julia Roeper ${ }^{1,2,3}$, Petros Christopoulos ${ }^{4,5} \wedge$, Markus Falk ${ }^{3,6} \wedge$, Lukas C. Heukamp ${ }^{3,6}$, Markus Tiemann ${ }^{3,6}$, \\ Albrecht Stenzinger ${ }^{7}$, Michael Thomas ${ }^{4,5} \wedge$, Frank Griesinger ${ }^{1,2,3}$ \\ ${ }^{1}$ Department of Internal Medicine-Oncology, Carl v. Ossietzky University of Oldenburg, Pius-Hospital, Oldenburg, Germany; ${ }^{2}$ Department of \\ Hematology and Oncology, Pius-Hospital, Oldenburg, Germany; ${ }^{3}$ Lung Cancer Network NOWEL Organisation, Pius-Hospital, Oldenburg, \\ Germany; ${ }^{4}$ Thoraxklinik and National Center for Tumor Diseases at Heidelberg University Hospital, Heidelberg, Germany; ${ }^{5}$ Translational Lung \\ Research Center Heidelberg (TLRC-H), member of the German Center for Lung Research (DZL), University Hospital, Heidelberg, Germany; \\ ${ }^{6}$ Hematopathology Hamburg, Hamburg, Germany; ${ }^{7}$ Department of General Pathology and Pathological Anatomy, University Hospital, Heidelberg, \\ Germany \\ Contributions: (I) Conception and design: F Griesinger, J Roeper; (II) Administrative support: F Griesinger, M Tiemann; (III) Provision of study \\ materials or patients: All authors; (IV) Collection and assembly of data: J Roeper, P Christopoulos; (V) Data analysis and interpretation: J Roeper, F \\ Griesinger, P Christopoulos, M Falk, M Thomas, A Stenzinger; (VI) Manuscript writing: All authors; (VII) Final approval of manuscript: All authors. \\ Correspondence to: Dr. Julia Roeper. Department of Hematology and Oncology, Pius-Hospital, Oldenburg, Georgstr. 12, 26121 Oldenburg, Germany. \\ Email: julia.roeper@uni-oldenburg.de.
}

Background: The negative prognostic and predictive value of TP53 co-mutations (TP53 mt+) in EGFR mutated (EGFR $\mathrm{mt}+)$ non-small cell lung cancer (NSCLC) is increasingly being acknowledged. Data consistently show that TP53 $\mathrm{mt}+$ impact negatively on 1st line objective response rate (ORR), progression free survival (PFS) and overall survival (OS) with 1st and 2nd generation tyrosine kinase inhibitors (TKI). However, a negative predictive impact has not been shown for the 3 rd generation TKI Osimertinib. Therefore, we investigated the impact of TP53 $\mathrm{mt}+$ in EGFR $\mathrm{mt}+$ NSCLC carrying a T790M resistance mutation and treated in 2nd/further lines with Osimertinib.

Methods: A total of 77 EGFR mt+ NSCLC IV patients carrying a T790M resistance mutation from two institutions were analyzed for TP53 $\mathrm{mt}$. Clinical data including sex, age, presence of CNS metastases, etc., as well as types of EGFR and TP53 $\mathrm{mt}+$ were captured. PFS and OS were calculated from the start of Osimertinib.

Results: TP53 $\mathrm{mt}+$ were found in $32 / 77$ patients $(42 \%)$. TP5 $3 \mathrm{mt}+$ was a statistically significant independent negative predictive factor for PFS and OS. PFS for TP53 mt+ patients were 9 months vs. 14 months for patients with TP53 wild-type (TP53WT) $(\mathrm{P}<0.008)$. OS for TP53 mt+ patients was 16 months vs. 24 months patients with TP53WT $(\mathrm{P}<0.025)$.

Conclusions: TP53 $\mathrm{mt}+$ have a negative impact on PFS and OS in a group of patients carrying a sensitizing EGFR $\mathrm{mt}+$ and a T790M resistance mutation treated with Osimertinib. These data, together with the data for 1 st/2nd generation TKI in 1 st line treatment call for additional therapeutic and management concepts for this subgroup of patients.

Keywords: Non-small cell lung cancer (NSCLC); metastatic; EGFR; TP53 co-mutation; Osimertinib

\footnotetext{
^ ORCID: Julia Roeper, 0000-0002-8660-7924; Petros Christopoulos, 0000-0002-7966-8980; Markus Falk, 0000-0002-4318-5039; Lukas

C. Heukamp, 0000-0002-3388-3482; Markus Tiemann, 0000-0003-4060-8355; Albrecht Stenzinger, 0000-0003-1001-103X; Michael Thomas, 0000-0001-9511-055X.
} 
Submitted Sep 14, 2021. Accepted for publication Dec 31, 2021.

doi: $10.21037 /$ tlcr-21-754

View this article at: https://dx.doi.org/10.21037/tlcr-21-754

\section{Introduction}

TP53 mutations are observed in wild-type (WT) non-smallcell lung cancer (NSCLC) with an incidence of about $50 \%$ and have been associated with smoking status (1). In EGFR mutated $(E G F R \mathrm{mt}+)$ NSCLC patients, the frequency of TP53 co-mutations (TP53 $\mathrm{mt}+$ ) ranges from $25.9 \%$ (2) to $49 \%$ (3-5) depending on the method of detection. TP53 encoding p 53 regulates cellular response to stress signals such as chemotherapy, radiation therapy and tyrosine kinase inhibition by inducing cell cycle arrest, senescence and/or apoptosis. Disruption of TP53's normal function can lead to malignant transformation. Since most chemotherapeutics and most likely tyrosine kinase inhibitors (TKI) induce DNA-damage and consequently activate $\mathrm{p} 53$, mutations in the TP53 gene might negatively affect response to cytotoxic treatment and thus represent a negative predictive factor (6-8). Also, TP53 mt+ might be a negative prognostic factor in lung cancer (9-12), as in other tumor types $(13,14)$.

The groups of Rosell, Crino and others have shown that TP53 $\mathrm{mt}+$ are negative predictors for chemotherapy or $1^{\text {st }}$ line TKI in EGFR $\mathrm{mt}+$ NSCLC. These analyses were hampered by inhomogeneous treatment or small numbers or the inclusion of different stages $(2-4,15)$. We have recently shown in a group of EGFR $\mathrm{mt}+$ NSCLC homogeneously treated in $1^{\text {st }}$ line therapy with $1^{\text {st }}$ or $2^{\text {nd }}$ generation TKI that TP53 $\mathrm{mt}+$ have a negative impact on the objective response rate (ORR), the progression free survival (PFS) and the overall survival (OS). We could show that TP53 $\mathrm{mt}+$ were not associated with specific clinical characteristics with one exception, i.e., the risk of developing central nervous system (CNS) metastases during the course of the disease. Also, TP $53 \mathrm{mt}+$ seemed to be stable during the course of the disease, as no new TP53 $\mathrm{mt}+$ were acquired at the time of $1^{\text {st }}$ or $2^{\text {nd }}$ generation EGFR TKI resistance (5).

With the FLAURA trial being positive for both PFS and OS in comparison to $1^{\text {st }}$ generation TKI, the standard of therapy for EGFR $\mathrm{mt}+\mathrm{NSCLC}$ has quickly changed to using $3^{\text {rd }}$ generation TKI in $1^{\text {st }}$ line therapy (16). Until now data on the potential impact of TP53 $\mathrm{mt}+$ in EGFR $\mathrm{mt}+$ NSCLC treated with Osimertinib are lacking. Therefore, we investigated the role of TP53 $\mathrm{mt}+$ in EGFR $\mathrm{mt}+\mathrm{NSCLC}$ carrying a T790M resistance mutation and being treated with Osimertinib. To this end a multicentric cohort of NSCLC patients treated at the University Hospitals of Oldenburg and Heidelberg was employed. As in $1^{\text {st }}$ and $2^{\text {nd }}$ generation TKIs, we demonstrated an important and statistically significant negative impact of TP53 $\mathrm{mt}+$ on PFS and OS.

We present the following article in accordance with the REMARK reporting checklist (available at https://tlcr. amegroups.com/article/view/10.21037/tlcr-21-754/rc).

\section{Methods}

\section{Patients}

To be included in this retrospective cohort study, patients had to be at least 18 years old and had to provide written consent to analyze their data for this project. All patients had to be tested for TP53 $\mathrm{mt}+$ at first diagnosis of EGFR positive NSCLC IV. Included patients were required to have a T790M resistance mutation against $1^{\text {st }}$ or $2^{\text {nd }}$ generation TKI, and have been treated with Osimertinib in the $2^{\text {nd }}$ or further therapy lines. Patients were excluded if they did not fulfill the inclusion criteria.

A total of 77 EGFR $\mathrm{mt}+$ NSCLC IV patients (76 Caucasians, 1 Asian) from two certified lung cancer centers from Germany (Pius Hospital Oldenburg; Thoraxklinik Heidelberg) were included in the study. All patients were $E G F R 3^{\text {rd }}$ generation TKI naïve when they started Osimertinib; 70/77 (91\%) patients were treated with TKI on $1^{\text {st }}$ line therapy and $7 / 77$ (9\%) with chemotherapy. In particular, patients who were treated with osimertinib in the further lines were initially treated with chemotherapy or had to be switched from one to another EGFR TKI due to toxicities.

The study was conducted in accordance with the Declaration of Helsinki (as revised in 2013). The study was approved by ethics committees of the University of Oldenburg (No. 2014-I) and the University of Heidelberg (No. S-145/2017). Written informed consent was obtained from all the participants. 


\section{Specimen characteristics}

Microtome sections $(5 \mu \mathrm{M})$ were prepared from FFPEtissue of NSCLC samples and one object slide was HE stained for tumor evaluation by a pathologist. Tumor tissue was gained from the remaining slides by manual microdissection, or in case material was limited, enriched by Laser Capture Microdissection (Leica CTR6500). DNA extraction was performed either manually (Macharey Nagel) or semi-automated (Maxwell MDx, Promega). The library preparation for the samples was performed using the Agilent SureSelect XT Kit as per the manufacturers' recommendations. Specimens were processed and stored at either Hematopathology Hamburg or at the Department of General Pathology and Pathological Anatomy, University Hospital Heidelberg.

\section{Assay methods}

Mutational analyses were performed by standard methods with hybrid capture assays as previously described (5). The Heidelberg cohort investigated for mutations by nextgeneration sequencing (NGS), as previously described (17). In all patients sufficient tumor material was available to analyze the TP53 status. No patient dropped out of the study. TP5 $3 \mathrm{mt}+$ were classified according to different algorithms as previously described (5). Further information is provided in the supplementary data. The interested reader can find them in a supplementary appendix online (Appendix 1).

\section{Study design}

The entire retrospective study data were obtained from the daily clinical setting, for this purpose the study does not require randomization, blinding of patients or coordinators. The 77 patients were first diagnosed at one of the two hospitals between 2011 and 2019. We followed up with patients until February 2021, median follow up calculated from start of Osimertinib was 21 months. In order to account for the influence of subsequent therapy after Osimertinib on OS, we captured the therapy after stop of Osimertinib: 4/77 (5\%) patients were treated after Osimertinib failure with I/O therapy $(\mathrm{n}=1)$, chemotherapy $(n=3)$ or TKI therapy $(n=3)$. Thus, the influence of subsequent therapy on OS is limited in this cohort.

Clinical and molecular data of the patients including sex, age, histology, smoking status, Eastern Cooperative
Oncology Group (ECOG) status, metastases at first diagnosis, presence of CNS metastases at baseline and during course of disease and comorbidities [Charlson Comorbidity Index (CCI)], as well as types of EGFR and TP53 $\mathrm{mt}+$ were captured. All clinical outcome parameters were calculated from the start of $3^{\text {rd }}$ generation TKI (ORR, PFS and OS).

\section{Statistical analysis}

The clinical characteristics of the 77 EGFR $\mathrm{mt}+$ NSCLC patients were descriptively evaluated.

Patient and tumor characteristics were compared using chi-square test or $t$-test of independence or Fisher exact test. To estimate the risk differences related to the TP53 status and the response to Osimertinib in $2^{\text {nd }}$ or further line therapy the odds ratio (OR) was calculated. For this purpose, a confidence interval of $95 \%$ was used, which corresponds to a significance level of $\mathrm{P}<0.05$. The correlations between patient characteristics and clinical outcome parameters PFS and OS are explored by non-parametric survival analyses using Kaplan-Meier calculations. The Kaplan-Meier method considered the different points of first diagnosis and the different observation periods of the patients during the analysis (18). In this retrospective analysis, bivariate dependencies of OS and PFS were shown with KaplanMeier curves and the log rank test for the calculation of $\mathrm{P}$ values. Furthermore, we constructed Cox regression models to predict the median PFS and the median OS separately in multivariate analyses. In the Cox regression models we used following covariates: histology, CNS at first diagnosis, CNS in front of Osimertinib, CNS after Osimertinib, CNS in total, age, sex, CCI, ECOG status, TP53 status, smoking status and EGFR exon status. Subsequently, the covariates were excluded step by step using the reverse procedure Forest. The results are presented as hazard ratio (HR) with $95 \%$ confidence intervals and $\mathrm{P}$ values.

\section{Results}

\section{Data}

Baseline characteristics of the 77 EGFR mt+ NSCLC IV patients are summarized in Table 1. All 77 (100\%) patients presented a common primary driver mutation, either del19 $(\mathrm{n}=58 / 77 ; 75 \%)$ or L858R ( $\mathrm{n}=19 / 77 ; 25 \%)$. The presence of EGFR $\mathrm{mt}+$ was associated with female sex $(\mathrm{n}=50 / 77$; $65 \%)$ and never/light smoking status $(\mathrm{n}=54 / 77 ; 71.4 \%)$. 
Table 1 Clinical characteristics of the EGFR mt+ NSCLC IV patients ( $\mathrm{n}=77)$

\begin{tabular}{|c|c|c|c|c|}
\hline Variable & TP53 $\mathrm{mt}+, \mathrm{n}=32$ & TP53WT, $\mathrm{n}=45$ & Total, $\mathrm{n}=77$ & $P$ value \\
\hline Sex & & & & 0.554 \\
\hline Male & $10(31 \%)$ & $17(38 \%)$ & $27(35 \%)$ & \\
\hline Female & $22(69 \%)$ & $28(62 \%)$ & $50(65 \%)$ & \\
\hline 0 & $17(53 \%)$ & $24(53 \%)$ & $41(53 \%)$ & \\
\hline 1 & $12(39 \%)$ & $17(38 \%)$ & $29(38 \%)$ & \\
\hline$\geq 2$ & $0(0 \%)$ & $1(2 \%)$ & $1(1 \%)$ & \\
\hline Histology & & & & 0.396 \\
\hline Metastases at first diagnosis & & & & 0.679 \\
\hline Brain & $7(22 \%)$ & $8(18 \%)$ & $15(19 \%)$ & \\
\hline Liver & $6(19 \%)$ & $6(13 \%)$ & $12(16 \%)$ & \\
\hline Other & 19 (59\%) & $31(69 \%)$ & $50(65 \%)$ & \\
\hline Smoking status & & & & 0.077 \\
\hline Never smoker & $20(40.8 \%)$ & $29(59.2 \%)$ & $49(63.6 \%)$ & \\
\hline Light smoker & $2(33.3 \%)$ & $4(66.7 \%)$ & $6(7.8 \%)$ & \\
\hline Current smoker & $5(29.4 \%)$ & $12(70.6 \%)$ & $17(22.1 \%)$ & \\
\hline At first diagnosis & $10(31 \%)$ & $12(27 \%)$ & $22(29 \%)$ & 0.661 \\
\hline Before Osimertinib & $15(47 \%)$ & $25(56 \%)$ & $40(52 \%)$ & 0.538 \\
\hline After Osimertinib & $18(56 \%)$ & $26(58 \%)$ & $44(57 \%)$ & 0.980 \\
\hline $\mathrm{CCl}$, mean (range) & $2.43(0-9)$ & $2.40(0-5)$ & $2.38(0-9)$ & 0.890 \\
\hline $\mathrm{CCl}$ groups & & & & 0.691 \\
\hline $0-2$ & $20(63 \%)$ & $26(58 \%)$ & $46(60 \%)$ & \\
\hline $3-4$ & $8(25 \%)$ & $15(33 \%)$ & $23(30 \%)$ & \\
\hline$\geq 5$ & $4(12 \%)$ & $4(9 \%)$ & $8(10 \%)$ & \\
\hline
\end{tabular}

In five patients no ECOG status was available; in one patient no smoking status was available, in one patient no CNS metastasis status could be detected before or after Osimertinib. EGFR mt+, epidermal growth factor receptor mutation; NSCLC, non-small cell lung cancer; TP53 mt+, tumor suppressor gene mutation; ECOG, Eastern Cooperative Oncology Groups; del19, deletion 19; L858R, exon 21 L858R mutation; CNS, central nervous system; CCI, Charlson Comorbidity Score. 


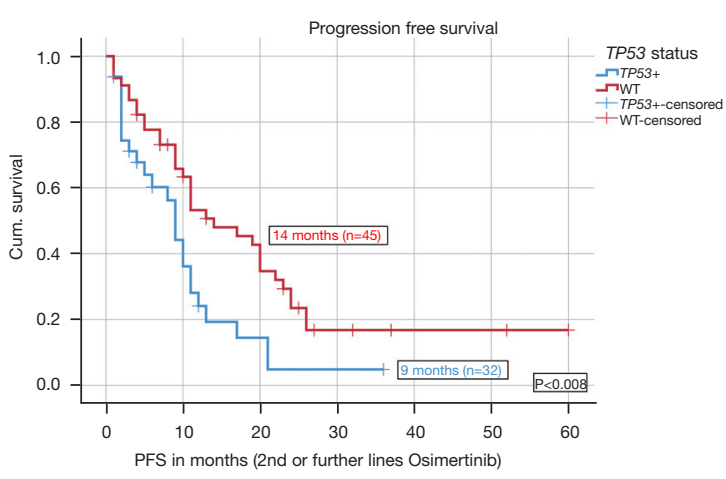

Figure 1 PFS of Osimertinib in 2nd/further lines depending on TP53 status. Kaplan-Meier curve on median PFS of Osimertinib in $2^{\text {nd }}$ or further line therapy for patients with TP53 $\mathrm{mt}+$ compared to patients with TP53WT; median PFS in months; for the calculation of the $\mathrm{P}$ value the $\log$ rank test was used. PFS, progression free survival; TP53, status tumor suppressor gene mutation status; WT, wild-type; cum., survival cumulative survival.

Median age was 64 years (range, 28-92 years). The vast majority of patients presented an ECOG status 0 or 1 $(\mathrm{n}=70 / 77 ; 91 \%)$. The average of the CCI was 2.38 (range, $0-9)$. A proportion of $28.6 \%(n=22 / 77)$ of patients had CNS metastases at primary diagnosis. Before start of Osimertinib, 18 patients had developed CNS metastases yielding to 40/77 (52\%) of patients with brain metastases. At progression of Osimertinib and discontinuation of Osimertinib, only four additional patients developed brain metastases resulting in a total of $44 / 77$ (57\%). Thirty-seven/77 (48\%) of the patients received Afatinib, 34/77 (44.1\%) Erlotinib and 6/77 (7.8\%) Gefitinib before start of Osimertinib. Seven patients ( $\mathrm{n}=7 / 77$, $9.1 \%$ ) had received chemotherapy in $1^{\text {st }}$ line therapy before being switched to $1^{\text {st }}$ or $2^{\text {nd }}$ generation TKI. All patients $(\mathrm{n}=77)$ were treated with Osimertinib in $2^{\text {nd }}(\mathrm{n}=51)$ or in further line therapy $(n=26)$ at occurrence of T790M.

All patients were studied for TP53 $\mathrm{mt}+$ at first diagnosis and $42 \%(\mathrm{n}=32 / 77)$ showed a TP53 $\mathrm{mt}+$. On TKI resistance $\left(1^{\text {st }}\right.$ or $2^{\text {nd }}$ generation TKI), all patients were rebiopsied (all with tissue). All patients had a T790M. In the Oldenburg cohort, all 11 patients with a TP53 $\mathrm{mt}+$ were retested for TP5 3 status. In $2 / 11$ (18\%) there were missing data for the TP 53 status. In 3/9 (33\%) patients, the TP53 reanalysis was not successful. In $6 / 6(100 \%)$ successfully retested patients, TP5 3 configuration was stable in comparison to the test before start of $1^{\text {st }}$ line TKI. In the Heidelberg cohort, the retest strategy for TP53 was different with comparison to the Oldenburg cohort. In Heidelberg, only patients with TP53WT were reevaluated at acquired resistance for TP53 status. Of the 38 TP53WT patients, 34 (89\%) were reevaluated at progression for TP53; 28/34 (82\%) patients were successfully retested for TP53. In 5/28 patients TP53 status changed at progression from WT to mutation.

Clinical characteristics were analyzed depending on the TP53 status and with the exception of age $(\mathrm{P}=0.042)$, no significant dependence as to any clinical characteristic was observed, including smoking status, type of EGFR $\mathrm{mt}+$ or CNS metastasis status. Table 1 shows the clinical characteristics of the patient cohort in total and stratified after the TP53 status.

\section{Analysis and presentation}

\section{ORR}

ORR was available in $72 / 77$ patients. ORR on Osimertinib in $2^{\text {nd }}$ or further lines was $45 \%(n=32 / 72), 39 \%(n=28 / 72)$ had a stable disease (SD) and $16.7 \%(n=12 / 72)$ of the patients had primary progression.

Stratified according to TP53 status, 38\% ( $\mathrm{n}=11 / 29)$ of patients with a TP53 $\mathrm{mt}+$ and $49 \%(\mathrm{n}=21 / 43)$ of patients with a TP53WT achieved an ORR [complete response $(\mathrm{CR}) /$ partial response $(\mathrm{PR})]$ on Osimertinib in $2^{\text {nd }}$ or further line therapy. Patients with a TP53WT constellation had a numerically higher chance to have an objective response to Osimertinib than patients with a TP53 $\mathrm{mt}+$ (OR 1.5620; $\mathrm{P}=0.362$ ) however this was not significantly different.

\section{PFS}

The PFS of $1^{\text {st }}$ line therapy with $1^{\text {st }}$ gen or $2^{\text {nd }}$ generation TKI was 13 months $(\mathrm{n}=31)$ for patients with TP53 mt+ and patients with TP53WT had an PFS of 17 months $(\mathrm{n}=39)(\mathrm{P}=0.249)$. In a homogenously TKI treated patient population in our center published in Oncotarget, there was a significant difference in ORR, PFS and OS in the EGFR $\mathrm{mt}+$ group dependent on TP53 status.

Patients with EGFR del19 mutations had a similar median PFS on Osimertinib in $2^{\text {nd }}$ and further line therapy [10 months $(\mathrm{n}=53)]$ as patients with EGFR L858R mutations [11 months ( $\mathrm{n}=19) ; \mathrm{P}=0.957$ ].

Stratified according to TP53 status, the median PFS on Osimertinib in $2^{\text {nd }}$ or further lines was significantly shorter (9 months, $\mathrm{n}=32$ ) for TP53 $\mathrm{mt}+$ patients compared to 14 months $(\mathrm{n}=45)$ for TP53WT patients $(\mathrm{P}=0.008)$. The HR for PFS on Osimertinib in $2^{\text {nd }}$ and further lines was 0.502 with a significant $P$ value of 0.012 . Figure 1 shows the median PFS on Osimertinib in $2^{\text {nd }}$ or further line therapy 


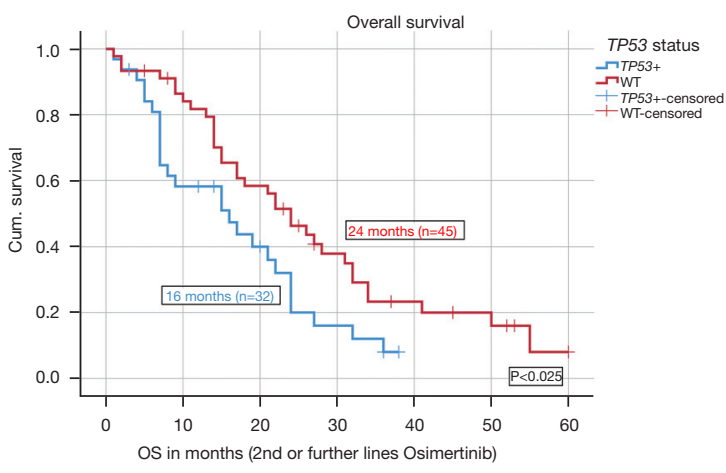

Figure 2 OS of Osimertinib in 2nd/further lines depending on TP53 status. Kaplan-Meier curve on median OS of Osimertinib in $2^{\text {nd }}$ or further line therapy for patients with TPS $3 \mathrm{mt}+$ compared to patients with TP53WT; median OS in months; for the calculation of the $\mathrm{P}$ value the log rank test was used. OS, overall survival; TP53, status tumor suppressor gene mutation status; WT, wildtype; cum., survival cumulative survival.

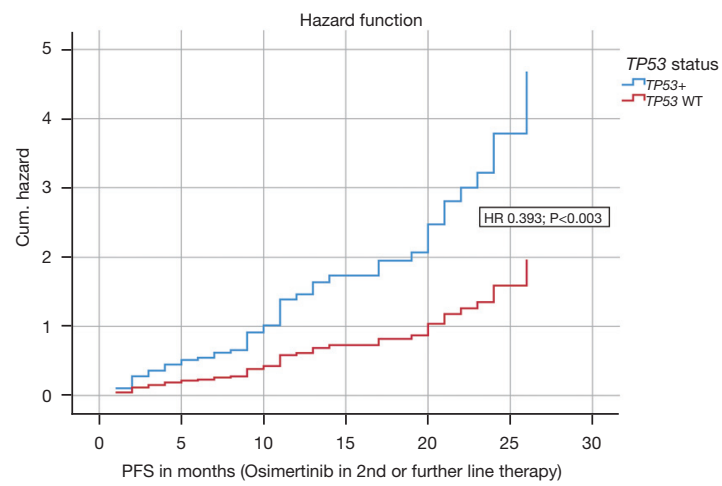

Figure 3 Hazard curve TP53 mt+ vs. TP53WT on PFS (Osimertinib in 2nd/further lines). The figure shows hazard curve for the TP53 status on median PFS on Osimertinib in $2^{\text {nd }}$ or further line therapy. In the cox regression model for PFS patients with a TP53WT had a $60.7 \%$ lower hazard of progression to Osimertinib in $2^{\text {nd }}$ or further line therapy than patients with a TP53 $\mathrm{mt}+$ PFS, progression free survival; TP53, status tumor suppressor gene mutation status; WT, wild-type; cum. hazard, cumulative hazard; $\mathrm{HR}$, hazard ratio.

in detail. More results including PFS data from different TP53 classifications and stratified after treatment lines are given in the Appendix 1. There were significant differences in PFS based on disruptive and nondisruptive, pathogenic and non-pathogenic mutations. Exon 8 was not significantly predictive due to low number (Tables S1,S2).

\section{OS}

The median OS for the entire cohort from the initial diagnosis was 46 months. Patients with EGFR del 19 mutations had a numerically longer median OS, calculated from the start of Osimertinib treatment in $2^{\text {nd }}$ and further line therapy [22 months $(\mathrm{n}=53)]$ compared to patients with L858R mutations [15 months $(\mathrm{n}=19)$; $\mathrm{P}=0.223]$, however the difference was not significant.

When stratified according to TP53 status, the median OS on Osimertinib in $2^{\text {nd }}$ or further lines was significantly shorter (16 months, $\mathrm{n}=32$ ) for TP53 $\mathrm{mt}+$ patients compared to 24 months for TP53WT patients $(\mathrm{n}=45)(\mathrm{P}=0.025)$. The HR for OS on Osimertinib in $2^{\text {nd }}$ and further lines was 0.561 with a significant $P$ value of 0.031 . Figure 2 shows the median OS of Osimertinib in $2^{\text {nd }}$ or further line therapy in detail. More results including OS data from different TP53 classifications and stratified after treatment lines are given in the Appendix 1. There were no significant differences in the OS based on disruptive and non-disruptive $\mathrm{mt}+$, pathogenic and non-pathogenic mt+ as well as exon 8 and non-exon 8 $\mathrm{mt}+$, most likely due to low numbers (Tables S3,S4).

\section{Multivariate analysis}

\section{PFS}

In a multivariate analysis for the endpoint PFS on Osimertinib in $2^{\text {nd }}$ or further line therapy the following covariates were included: CNS metastases at first diagnosis, CNS metastases after Osimertinib, CNS metastases in total, sex, CCI, ECOG status, TP53 status, smoking status, EGFR exon status. The model with the 9 variables was statistically significant $\{$ likelihood $=380.694$; Chi-square [9] $=17.386$; $\mathrm{P}=0.043\}$. The covariates TP53 status (HR 0.393; $\mathrm{P}=0.003$ ) and ECOG status (HR 0.174; $\mathrm{P}=0.045$ ) contributed significantly to the survival time model. Figure 3 shows the hazard curve of the covariate TP53 status and its negative impact on PFS (Osimertinib in $2^{\text {nd }}$ or further line therapy). Table 2 presents the survival time model and the covariates in detail.

\section{OS}

In a multivariate analysis on the endpoint OS on Osimertinib in 2 nd or further line therapy the following covariates were included: histology, CNS metastases at first diagnosis, CNS metastases before Osimertinib, CNS metastases after Osimertinib, CNS metastases in total, age, sex, CCI, ECOG status, TP53 status, smoking status, EGFR exon status. The model with the 12 covariates was 
Table 2 Multivariate analysis on PFS and OS

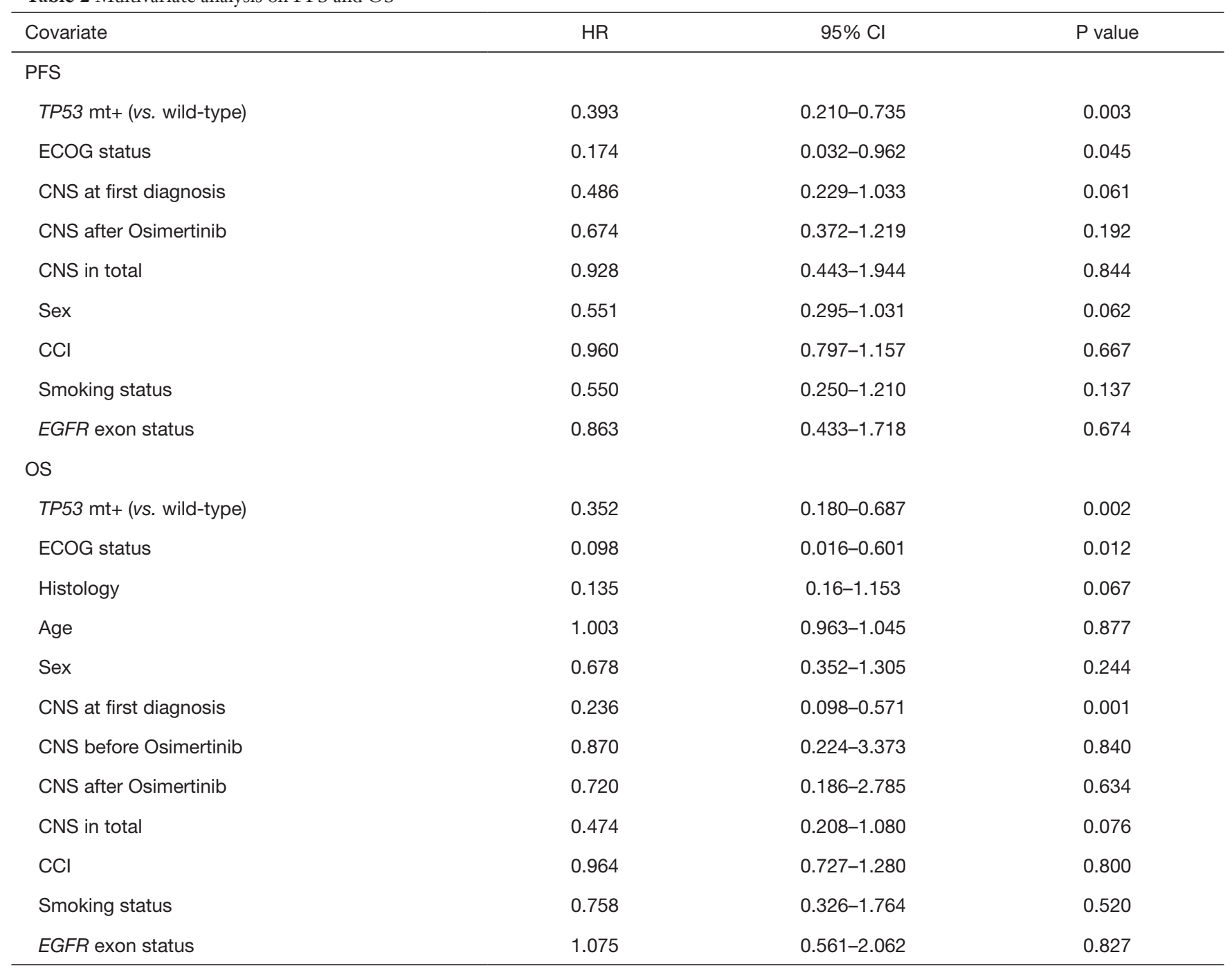

PFS, progression free survival; OS, overall survival; HR, hazard ratio; Cl, confidence interval; TP53 mt+, tumor suppressor gene; ECOG, Eastern Cooperative Oncology Groups; CNS, central nervous system; CCl, Charlson Comorbidity Score; EGFR, epidermal growth factor receptor.

statistically significant $\{$ likelihood $=388.239$; Chi-square[12] $=23.144 ; \mathrm{P}=0.027\}$. The covariates TP53 status $(\mathrm{HR} 0.352$; $\mathrm{P}=0.002$ ), ECOG status (HR 0.098; $\mathrm{P}=0.012$ ) and $\mathrm{CNS}$ at first diagnosis (HR 0.236; $\mathrm{P}=0.001$ ) contributed significantly to the survival time model and were independent negative factors. Figure 4 shows the hazard curve of the covariate TP53 status and its negative impact on OS (Osimertinib in $2^{\text {nd }}$ or further line therapy). Table 2 presents the survival time model and the other covariates in detail.

\section{Discussion}

Previous studies showed that TP53 $\mathrm{mt}+$ have a negative impact on ORR, PFS and OS in EGFR $\mathrm{mt}+$ NSCLC treated in $1^{\text {st }}$ line with $1^{\text {st }}$ or $2^{\text {nd }}$ generation EGFR TKI $(1-5,14,15-19)$. The current study addressed the question whether TP53 $\mathrm{mt}+$ influences the outcome when patients are treated with Osimertinib, a $3^{\text {rd }}$ generation EGFR TKI that was initially approved for $2^{\text {nd }}$ line treatment of EGFR $\mathrm{mt}+$ NSCLC patients carrying a T790M resistance mutation. 


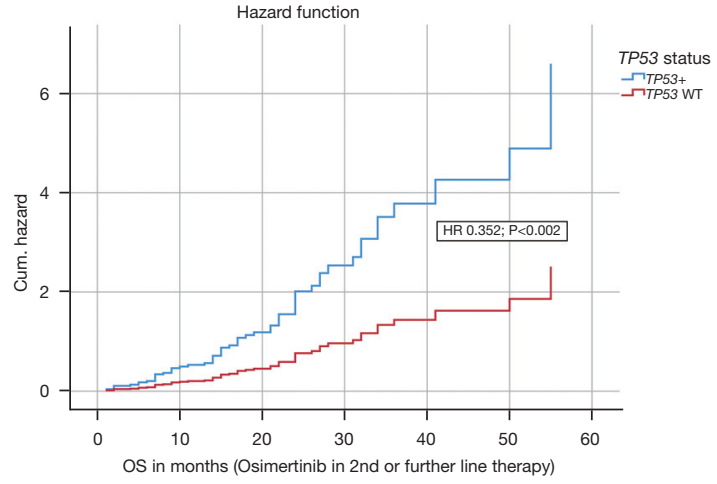

Figure 4 Hazard curve TP53 $\mathrm{mt}+v s$. TP53W'T on OS (Osimertinib in $2 \mathrm{nd}$ /further lines). The figure shows the hazard curve for the TP53 status on median OS on Osimertinib in $2^{\text {nd }}$ or further line therapy. In the cox regression model for OS patients with a TP53WT had a $64.8 \%$ lower hazard of progression to Osimertinib in $2^{\text {nd }}$ or further line therapy than patients with a TP5 $3 \mathrm{mt}+$. OS, overall survival; TP53, status tumor suppressor gene mutation status; WT, wild-type; cum., hazard cumulative hazard; HR, hazard ratio.

Our data show that the presence of TP53 mt+ impact ORR (not significant) and PFS and OS significantly. Overall, the low response rate of $45 \%$ for Osimertinib in our cohort and the significant difference in OS between del19 and L858R (Table S3) is somewhat different to the results of the AURA3 trial $(19,20)$. As our study is a retrospective study there is a possibility of group selection bias due to the small sample size. Therefore, there might be a limitation as to the association between TP53 $\mathrm{mt}+$ and the effect of TKIs in our analysis.

The prevalence/frequency of TP53 $\mathrm{mt}+$ was similar in this group of homogeneously EGFR T790M positive patients compared to a group of patients analyzed before the start of $1^{\text {st }}$ line therapy, underlining the fact that TP53 $\mathrm{mt}+$ seem to be stable throughout the course of the disease. Notably, with the exception of a weak association with age, TP53 $\mathrm{mt}+$ was not associated with any clinical parameters such as sex and smoking status. There was a slightly higher incidence of CNS metastases at baseline (before the start of Osimertinib) in the TP53 $\mathrm{mt}+$ subgroup compared to the TP53WT group, consistent with the finding that TP53 $\mathrm{mt}+$ confer a higher risk of progression in the CNS on $1^{\text {st }}$ or $2^{\text {nd }}$ generation TKI (5). Importantly CNS progression on Osimertinib was not different in the TP5 $3 \mathrm{mt}+$ compared to the TP53WT group consistent with the high CNS efficacy of Osimertinib in the brain.

TP53 $\mathrm{mt}+$ were significantly associated with inferior outcome both in univariate as well as multivariate analyses. Therefore, the impact of TP53 $\mathrm{mt}+$ seems to be consistent independently of the generation of EGFR TKI. It is difficult to imagine why Osimertinib would overcome the effect of TP53 $\mathrm{mt}+$ in EGFR $\mathrm{mt}+\mathrm{NSCLC}$, as the mechanism of action is dependent on the inhibitor of the EGFR pathway as with 1 st and 2 nd generation TKI.

Similarly, in $A L K$ translocated NSCLC, it is well known that TP53 $\mathrm{mt}+$ impact PFS and OS, an effect which is independent of the $A L K$ fusion variant $(21,22)$. In a recent analysis with the $3^{\text {rd }}$ generation $A L K$ inhibitor Lorlatinib, it was shown that TP53 $\mathrm{mt}+$ impact significantly on PFS and OS therefore underlining the overriding effect of TP53 $\mathrm{mt}+$ on the effectiveness of the $3^{\text {rd }}$ generation $A L K$ inhibitor (23).

Given the negative impact of TP53 mt+ irrespective of the line of treatment and irrespective of the generation of EGFR TKI, it is tempting to speculate that $1^{\text {st }}$ line Osimertinib might also be less efficacious in EGFR $\mathrm{mt}+$ patients carrying a TP53 $\mathrm{mt}+$. An analysis of the TP53 $\mathrm{mt}+$ analysis within the FLAURA trial and their impact on PFS and OS are eagerly awaited.

In our opinion, it is clear that these data should have an impact on the design of future trials, i.e., that future $E G F R$ $\mathrm{mt}+$ trials should be stratified for the presence of TP53 $\mathrm{mt}+$ This strategy would help to dissect the impact of TP53 $\mathrm{mt}+$ on ORR, PFS and OS in a controlled trial scenario. Second, with the revival of TKI and chemotherapy combinations, it would be of interest to investigate whether the influence of TP5 $3 \mathrm{mt}+$ might be overcome by the adding chemotherapy to EGFR TKI. Third, in routine practice we would advocate for testing patients for TP53 $\mathrm{mt}+$ and for monitoring these patients more closely when treated with TKI therapy then patients with TP53WT.

Sallman et al. demonstrated that combination treatment with a TP53 modulator, that restores TP53 function when mutated or inactivated (APR-246) and chemotherapy (Azacytidine) was well-tolerated yielding high rates of clinical response $(71 \%)$ and an OS of 10.8 months in patients with TP53-mutant myelodysplastic syndrome (MDS) and oligoblastic acute myeloid leukemia (AML) (24). Finally, as TP53 modulators, such as APR-246 have been shown to be effective in hematologic disorders, one could envision to combine EGFR TKI and TP53 modulators in NSCLC clinical trials. 


\section{Conclusions}

As in $1^{\text {st }}$ line therapy with $1^{\text {st }}$ and $2^{\text {nd }}$ generation TKI, TP53 $m t+$ have a negative impact on ORR, PFS and OS in $2^{\text {nd }}$ and further line with the $3^{\text {rd }}$ generation TKI Osimertinib. Strategies should be developed to monitor TP53 $\mathrm{mt}+$ patients on EGFR TKI more closely than TP53WT patients. Clinical studies should stratify for the presence of TP53 $\mathrm{mt}+$ and should investigate EGFR TKI combinations, potentially with chemotherapy or with specific TP53 modulators.

\section{Acknowledgments}

Thanks to all patients and their families for generating the data to enable this study.

Funding: None.

\section{Footnote}

Reporting Checklist: The authors have completed the REMARK reporting checklist. Available at https://tlcr. amegroups.com/article/view/10.21037/tlcr-21-754/rc

Data Sharing Statement: Available at https://tlcr.amegroups. com/article/view/10.21037/tlcr-21-754/dss

Peer Review File: Available at https://tlcr.amegroups.com/ article/view/10.21037/tlcr-21-754/prf

Conflicts of Interest: All authors have completed the ICMJE uniform disclosure form (available at https://tlcr. amegroups.com/article/view/10.21037/tlcr-21-754/coif). JR reports speaker's honoraria: AstraZeneca, Boehringer Ingelheim. PC reports advisory board/speaker's honoraria: AstraZeneca, Boehringer Ingelheim, Roche; research grants: AstraZeneca, Roche. MF reports advisory board: Boehringer Ingelheim, Pfizer; speaker's honoraria: Roche, AstraZeneca, Boehringer Ingelheim. LCH reports advisory board/speaker's honoraria: AstraZeneca, Boehringer Ingelheim, BMS, Pfizer, MSD, Novartis, Roche, Siemens. M Tiemann reports speaker's honoraria: AstraZeneca, Boehringer Ingelheim. AS reports advisory board/speaker's bureau: AIgnostics, Astra Zeneca, AGCT, Bayer, BMS, Eli Lilly, Illumina, Janssen, MSD, Novartis, Pfizer, Roche, Seattle Genetics, Takeda, Thermo Fisher; travel/research grants: Bayer, BMS, Chugai. M Thomas advisory board/ speaker's honoraria: AbbVie, AstraZeneca, Amgen, BMS,
Boehringer Ingelheim, Celgene, Chugai, Janssen, Lilly, MSD, Merck, Novartis, Pfizer, Roche, Takeda; travel/ research grants: AbbVie, AstraZeneca, BMS, Boehringer Ingelheim, Celgene, Chugai, Lilly, MSD, Novartis, Pfizer, Roche, Takeda. FG reports advisory board/speaker's honoraria: AstraZeneca, Boehringer Ingelheim, BMS, Celgene, Lilly, MSD, Novartis, Pfizer, Roche, Takeda, Ariad, Abbvie, Siemens; research grants: AstraZeneca, Boehringer Ingelheim, BMS, Celgene, Lilly, MSD, Novartis, Pfizer, Roche, Takeda, Siemens. The authors have no other conflicts of interest to declare.

Ethical Statement: The authors are accountable for all aspects of the work in ensuring that questions related to the accuracy or integrity of any part of the work are appropriately investigated and resolved. The study was conducted in accordance with the Declaration of Helsinki (as revised in 2013). The study was approved by ethics committees of the University of Oldenburg (No. 2014I) and the University of Heidelberg (No. S-145/2017). Written informed consent was obtained from all the participants.

Open Access Statement: This is an Open Access article distributed in accordance with the Creative Commons Attribution-NonCommercial-NoDerivs 4.0 International License (CC BY-NC-ND 4.0), which permits the noncommercial replication and distribution of the article with the strict proviso that no changes or edits are made and the original work is properly cited (including links to both the formal publication through the relevant DOI and the license). See: https://creativecommons.org/licenses/by-nc-nd/4.0/.

\section{References}

1. Gu J, Zhou Y, Huang L, et al. TP53 mutation is associated with a poor clinical outcome for non-small cell lung cancer: Evidence from a meta-analysis. Mol Clin Oncol 2016;5:705-13.

2. Molina-Vila MA, Bertran-Alamillo J, Gascó A, et al. Nondisruptive $\mathrm{p} 53$ mutations are associated with shorter survival in patients with advanced non-small cell lung cancer. Clin Cancer Res 2014;20:4647-59.

3. Canale M, Petracci E, Delmonte A, et al. Impact of TP53 Mutations on Outcome in EGFR-Mutated Patients Treated with First-Line Tyrosine Kinase Inhibitors. Clin Cancer Res 2017;23:2195-202.

4. Labbé C, Cabanero M, Korpanty GJ, et al. Prognostic and 
predictive effects of TP53 co-mutation in patients with EGFR-mutated non-small cell lung cancer (NSCLC). Lung Cancer 2017;111:23-9.

5. Roeper J, Falk M, Chalaris-Rißmann A, et al. TP53 comutations in EGFR mutated patients in NSCLC stage IV: A strong predictive factor of ORR, PFS and OS in EGFR mt+ NSCLC. Oncotarget 2020;11:250-64.

6. Viktorsson K, De Petris L, Lewensohn R. The role of p53 in treatment responses of lung cancer. Biochem Biophys Res Commun 2005;331:868-80.

7. Wattel E, Preudhomme C, Hecquet B, et al. p53 mutations are associated with resistance to chemotherapy and short survival in hematologic malignancies. Blood 1994;84:3148-57.

8. Reles A, Wen WH, Schmider A, et al. Correlation of p53 mutations with resistance to platinum-based chemotherapy and shortened survival in ovarian cancer. Clin Cancer Res 2001;7:2984-97.

9. Mogi A, Kuwano H. TP53 mutations in nonsmall cell lung cancer. J Biomed Biotechnol 2011;2011:583929.

10. Mitsudomi T, Hamajima N, Ogawa M, et al. Prognostic significance of $\mathrm{p} 53$ alterations in patients with nonsmall cell lung cancer: a meta-analysis. Clin Cancer Res 2000;6:4055-63.

11. Steels E, Paesmans M, Berghmans T, et al. Role of p53 as a prognostic factor for survival in lung cancer: a systematic review of the literature with a meta-analysis. Eur Respir J 2001;18:705-19.

12. Ahrendt SA, Hu Y, Buta M, et al. p53 mutations and survival in stage I non-small-cell lung cancer: results of a prospective study. J Natl Cancer Inst 2003;95:961-70.

13. Döhner H, Stilgenbauer S, Benner A, et al. Genomic aberrations and survival in chronic lymphocytic leukemia. N Engl J Med 2000;343:1910-6.

14. Poeta ML, Manola J, Goldwasser MA, et al. TP53 mutations and survival in squamous-cell carcinoma of the head and neck. N Engl J Med 2007;357:2552-61.

15. Kron A, Alidousty C, Scheffler M, et al. Impact of TP53

Cite this article as: Roeper J, Christopoulos $\mathrm{P}$, Falk M, Heukamp LC, Tiemann M, Stenzinger A, Thomas M, Griesinger F. TP53 co-mutations as an independent prognostic factor in 2nd and further line therapy-EGFR mutated nonsmall cell lung cancer IV patients treated with osimertinib. Transl Lung Cancer Res 2022;11(1):4-13. doi: 10.21037/tlcr21-754 mutation status on systemic treatment outcome in ALKrearranged non-small-cell lung cancer. Ann Oncol 2018;29:2068-75.

16. Ramalingam SS, Vansteenkiste J, Planchard D, et al. Overall Survival with Osimertinib in Untreated, EGFR-Mutated Advanced NSCLC. N Engl J Med 2020;382:41-50.

17. Volckmar AL, Leichsenring J, Kirchner M, et al. Combined targeted DNA and RNA sequencing of advanced NSCLC in routine molecular diagnostics: Analysis of the first 3,000 Heidelberg cases. Int J Cancer 2019;145:649-61.

18. Zwiener I, Blettner M, Hommel G. Survival analysis: part 15 of a series on evaluation of scientific publications. Dtsch Arztebl Int 2011;108:163-9.

19. Mok TS, Wu YL, Ahn MJ, et al. Osimertinib or PlatinumPemetrexed in EGFR T790M-Positive Lung Cancer. N Engl J Med 2017;376:629-40.

20. Papadimitrakopoulou VA, Mok TS, Han JY, et al. Osimertinib versus platinum-pemetrexed for patients with EGFR T790M advanced NSCLC and progression on a prior EGFR-tyrosine kinase inhibitor: AURA3 overall survival analysis. Ann Oncol 2020;31:1536-44.

21. Christopoulos P, Kirchner M, Roeper J, et al. Risk stratification of EGFR+ lung cancer diagnosed with panel-based next-generation sequencing. Lung Cancer 2020;148:105-12.

22. Christopoulos P, Kirchner M, Bozorgmehr F, et al. Identification of a highly lethal V3+ TP53+ subset in ALK+ lung adenocarcinoma. Int J Cancer 2019;144:190-9.

23. Frost N, Christopoulos P, Kauffmann-Guerrero D, et al. Lorlatinib in pretreated ALK- or ROS1-positive lung cancer and impact of TP53 co-mutations: results from the German early access program. Ther Adv Med Oncol 2021;13:1758835920980558.

24. Sallman DA, DeZern AE, Garcia-Manero G, et al. Eprenetapopt (APR-246) and Azacitidine in TP53Mutant Myelodysplastic Syndromes. J Clin Oncol 2021;39:1584-94. 


\section{Details on the detection of TP53 co-mutations and the three different TP53 classifications}

\section{Assay methods}

Against the background of technological advances in recent years, EGFR exon 18-21 and TP53 (exons 4-10) mutational analysis were performed by NGS-based methods. Alternatively, an amplicon-based NGS panel (Illumina platform) was used to detect mutations in 38-42 relevant genes, including TP53. Part of the samples were analyzed with a hybrid capture based target enrichment followed by massively parallel sequencing [Hybrid Capture NGS, NeoSelect, NEO New Oncology, IonTorrent (ThermoFisher Scientific)]. The library preparation for the samples was performed using the Agilent SureSelect XT Kit as per the manufacturers' recommendations $(5,17)$.

TP53 $\mathrm{mt}+$ were classified according to three different algorithms as previously described: (I) classification by Poeta et al. (14), (II) by an extended algorithm based on Poeta et al. (14) with additional parameters like structural prediction and GVDV biophysical analysis (25) and (III) based on exon 8 vs. non-exon 8 mutations (4).

In an effort to specify the functional significance of the respective mutations in further detail (14), we included additional parameters in order to modify differentiation into pathogenic vs. non-pathogenic TP53 co-mutations (25). These mutations are likely to interfere with TP53 function significantly. Also, if an Align-GVGD score of C65 was reached, mutations were classified as pathogenic. Specifically, DNA-contact-mutations R273C, R273G, R248Q were reclassified as pathogenic mutations, since functional impairment is likely (25). Mutation R280I is located within the LSH2- (loop-sheet-helix region 2), which is part of the DNA-binding core and was therefore re-categorized as pathogenic. Mutations H179R and C176S constitute $\mathrm{Zn}^{2+}$-binding sites and were therefore also regarded as pathogenic upon review.

The third classification was recently proposed by the group of Canale et al. (3). The authors characterized a cohort of EGFR $\mathrm{mt}+$ patients that in $30.1 \%$ of cases carried additional TP53 $\mathrm{mt}+$ and these were categorized based on exons. TP $33 \mathrm{mt}+$ within exon 8 were associated with significantly lower DCR, and shorter PFS and OS. In addition to that, we showed similar results for TP53 exon 8 co-mutations in our EGFR mt+ NSCLC IV cohort treated with 1st or 2nd generation TKI's.

Detailed results on each treatment line with Osimertinib and the three different TP53 classifications.

\section{Results}

Analysis and presentation

PFS

Table S1 Median PFS in months on Osimertinib in 2nd line therapy

\begin{tabular}{|c|c|c|c|}
\hline & $\mathrm{n}$ & PFS & $P$ value \\
\hline EGFR exon status & & & 0.684 \\
\hline del19 & 33 & 10 & \\
\hline L858R & 15 & 11 & \\
\hline TP53 status & & & 0.033 \\
\hline TP53 mt+ & 24 & 13 & \\
\hline TP53WT & 27 & 9 & \\
\hline TP53 status according to Poeta et al. (14) & & & 0.100 \\
\hline TP53 disruptive mt+ & 15 & 8 & \\
\hline TP53 non-disruptive $\mathrm{mt}+$ & 9 & 11 & \\
\hline TP53WT & 27 & 13 & \\
\hline TP53 status according to Roeper et al. (25) & & & 0.079 \\
\hline TP53 pathogenic $\mathrm{mt}+$ & 17 & 8 & \\
\hline TP53 non-pathogenic mt+ & 7 & 12 & \\
\hline TP53WT & 27 & 13 & \\
\hline TP53 status according to Canale et al. (3) & & & 0.052 \\
\hline TP53 exon 8 & 4 & 10 & \\
\hline TP53 non-exon 8 & 20 & 8 & \\
\hline TP53WT & 27 & 13 & \\
\hline
\end{tabular}

PFS, progression free survival; EGFR, epidermal growth factor receptor; del19, deletion 19; L858R, exon 21 L858R mutation; TP53, status tumor suppressor gene status; TP53 mt+, tumor suppressor gene mutation; WT, wild-type; mt+, mutation. 
Table S2 Median PFS in months on Osimertinib in 2nd and further line therapy

\begin{tabular}{|c|c|c|c|}
\hline & $\mathrm{n}$ & PFS & $P$ value \\
\hline TP53 status according to Poeta et al. (14) & & & 0.011 \\
\hline TP53 disruptive $\mathrm{mt}+$ & 19 & 8 & \\
\hline TP53 non-disruptive $\mathrm{mt}+$ & 13 & 11 & \\
\hline TP53WT & 45 & 14 & \\
\hline TP53 status according to Roeper et al. (25) & & & 0.030 \\
\hline TP53 pathogenic $\mathrm{mt}+$ & 23 & 9 & \\
\hline TP53 non-pathogenic $\mathrm{mt}+$ & 9 & 11 & \\
\hline TP53WT & 45 & 14 & \\
\hline TP53 status according to Canale et al. (3) & & & 0.017 \\
\hline TP53 exon 8 & 4 & 10 & \\
\hline TP53 non-exon 8 & 28 & 9 & \\
\hline TP53WT & 45 & 14 & \\
\hline
\end{tabular}

PFS, progression free survival; TP53, tumor suppressor gene status; mt+, mutation; TP53 mt+, tumor suppressor gene mutation; WT, wildtype.

\section{OS}

Table S3 Median OS in months on Osimertinib in 2nd line therapy

\begin{tabular}{|c|c|c|c|}
\hline & $\mathrm{n}$ & OS & $P$ value \\
\hline EGFR exon status & & & 0.019 \\
\hline del19 & 33 & 24 & \\
\hline L858R & 15 & 11 & \\
\hline TP53 status & & & 0.135 \\
\hline TP53 mt+ & 24 & 16 & \\
\hline TP53WT & 27 & 24 & \\
\hline TP53 status according to Poeta et al. (14) & & & 0.287 \\
\hline TP53 disruptive $\mathrm{mt}+$ & 15 & 21 & \\
\hline TP53 non-disruptive $\mathrm{mt}+$ & 9 & 15 & \\
\hline TP53WT & 27 & 24 & \\
\hline TP53 status according to Roeper et al. (25) & & & 0.250 \\
\hline TP53 pathogenic $\mathrm{mt}+$ & 17 & 21 & \\
\hline TP53 non-pathogenic $\mathrm{mt}+$ & 7 & 15 & \\
\hline TP53WT & 27 & 24 & \\
\hline TP53 status according to Canale et al. (3) & & & 0.232 \\
\hline TP53 exon 8 & 4 & 27 & \\
\hline TP53 non-exon 8 & 20 & 15 & \\
\hline TP53WT & 27 & 24 & \\
\hline TP53WT & 27 & 13 & \\
\hline
\end{tabular}

OS, overall survival; EGFR, epidermal growth factor receptor; del19, deletion 19; L858R, exon 21 L858R mutation; TP53, status tumor suppressor gene status; TP53 mt+, tumor suppressor gene mutation; WT, wild-type; mt+, mutation. 
Table S4 Median OS in months on Osimertinib in 2nd and further line therapy

\begin{tabular}{|c|c|c|c|}
\hline & $\mathrm{n}$ & OS & $P$ value \\
\hline TP53 status according to Poeta et al. (14) & & & 0.081 \\
\hline TP53 disruptive $\mathrm{mt}+$ & 19 & 16 & \\
\hline TP53 non-disruptive $\mathrm{mt}+$ & 13 & 15 & \\
\hline TP53WT & 45 & 24 & \\
\hline TP53 status according to Roeper et al. (25) & & & 0.032 \\
\hline TP53 pathogenic $\mathrm{mt}+$ & 23 & 16 & \\
\hline TP53 non-pathogenic mt+ & 9 & 15 & \\
\hline TP53WT & 45 & 24 & \\
\hline TP53 status according to Canale et al. (3) & & & 0.054 \\
\hline TP53 exon 8 & 4 & 27 & \\
\hline TP53 non-exon 8 & 28 & 15 & \\
\hline TP53WT & 45 & 24 & \\
\hline
\end{tabular}

OS, overall survival; TP53, status tumor suppressor gene status; TP53 mt+, tumor suppressor gene mutation; mt+, mutation; WT, wild-type.

\section{References}

25. Joerger AC, Fersht AR. Structure-function-rescue: the diverse nature of common p 53 cancer mutants. Oncogene 2007;26:2226-42. 